\title{
Characterization of hexadecyltrimethylammonium-organoclay and its individual components by thermal techniques
}

\author{
PABLO M NARANJO ${ }^{1, *}$, EDGARDO L SHAM ${ }^{1,2}$ and ELSA M FARFÁN TORRES ${ }^{1,3}$ \\ ${ }^{1}$ INIQUI-CONICET (Instituto de Investigaciones para la Industria Química-Consejo Nacional de Investigaciones \\ Científicas y Técnicas), A4408FVY Salta, Argentina \\ ${ }^{2}$ Fac. Ingeniería, Universidad Nacional de Salta, A4408FVY Salta, Argentina \\ ${ }^{3}$ Fac. Cs. Exactas, Universidad Nacional de Salta, A4408FVY Salta, Argentina \\ *Author for correspondence (pmnaranjo@gmail.com; pnaranjo@unsa.edu.ar)
}

MS received 19 November 2015; accepted 16 September 2016; published online 26 July 2017

\begin{abstract}
The properties of the materials determine their potential applications. The aim of this article is to study the properties of the organoclays using simple and rapid technologies. Organoclays with different surfactant loadings (SL) were synthesized using an Argentine bentonite with a high content of montmorillonite (Bent) and hexadecyltrimethylammonium bromide as cationic surfactant. The samples were characterized using thermal techniques. The results revealed that the hydrophilicity of the organoclays decreases with increasing SL until the SL reaches 0.8 times the cation exchange capacity of the clay; and remains constant at a higher surfactant load. The stability of organoclays was inversely proportional to the SL of each sample. The layers showed a stabilization of approximately $40^{\circ} \mathrm{C}$ for their structural transformation temperature, caused by the presence of the surfactant. In addition, at a SL $<1.0$ the surfactant presented a 'liquid-like' structure in the interlayer space, whereas at a SL $>1.0$ the structure was 'solid-like'.
\end{abstract}

Keywords. Cationic surfactant; montmorillonite; organoclay; thermal techniques.

\section{Introduction}

Recently, organoclays have been widely studied because of their great academic and technological interest [1-3]. This material is obtained by interaction between a clay and a generally cationic, organic component. The most commonly used clays in the synthesis of organoclays are smectites and within this group, montmorillonites.

In general, the presence of organic components grants the material organophylic characteristics [4-7] and this is one of the most important properties. Although some studies have reported an increase in hydrophilicity [8-10], these have been very particular cases.

The main applications of these materials arise because of the increase in organophylicity of the organoclays compared with the original clay. Some of the most common applications are adsorption of organic and anionic components in aqueous solution [11-16] and synthesis of polymer nanocomposites [17-19]. In the latter case one of the desired effects is that the presence of organoclay generates an increase in the thermal stability of the polymer.

The principal components of an organoclay sample are: cationic surfactants, clay layers and water molecules. Caused by the notable difference in the properties of these components, it is relatively easy to identify independently the data corresponding to each component.
$\mathrm{X}$-ray diffraction (XRD), Fourier transform infrared spectroscopy (FTIR), X-ray photoelectron spectroscopy, thermogravimetric analysis (TG), thermal differential analysis (DTA) and differential scanning calorimetry (DSC) techniques are widely used in the characterization of organoclays [3,20-26]. Particularly the thermal techniques provide a wealth of information [5,27-31].

In this study the characterization of organoclays, with special emphasis on the behaviour of the individual components, is presented. The behaviour of the clay layers, the cationic surfactant and the water molecules present in the material were assayed using TG, DTA and DSC thermal techniques. On the basis of the information provided by these studies, organoclays with specific properties can be designed according to their application.

\section{Materials and methods}

\subsection{Materials}

Natural Castiglione clay, mainly composed of montmorillonite (84\% montmorillonite, $12 \%$ feldspar and $4 \%$ quartz), was used as starting material. This material presented a cation exchange capacity (CEC) of $83.7 \mathrm{mmol}$ per $100 \mathrm{~g}$ and it was determined by using the $\mathrm{Cu}(\mathrm{EDA})_{2}^{2+}$ technique [32]. 
Hexadecyltrimethylammonium bromide (Br-HDTMA), was purchased from Merck (Argentina), was used as a cationic surfactant. All solutions were prepared using deionized water obtained through inverse osmosis using an SQC 3 Reverse Osmosis system from 3M Purification Inc.

\subsection{Preparation of organoclays}

Organoclays were prepared with a surfactant loading (SL) between 0.2 and 4.0 times the CEC. Preparations were carried out in batches by bringing $50 \mathrm{ml}$ of a suspension of approximately $3 \% \mathrm{w} / \mathrm{v}$ of the original clay in contact with different volumes of a $5 \times 10^{-3}$ M HDTMA solution at a final volume of $500 \mathrm{ml}$. This suspension was agitated for $2 \mathrm{~h}$ without controlled temperature. Later, the solid fraction (organoclay) was separated from the supernatant fraction by centrifugation at 10,000 rpm. The solid fraction was washed with distilled water until complete elimination of the $\mathrm{Br}^{-}$ (this was confirmed by testing negative with $\mathrm{AgNO}_{3}$ solution). The different organoclays thus obtained were dried at $60^{\circ} \mathrm{C}$, ground and kept in a desiccator for posterior characterization; samples were expressed as Bent, followed by the SL (Bent-0.4, Bent-0.8, etc.).

\subsection{Characterization of the samples}

Thermogravimetric analysis and DTA were carried out using a Rigaku TAS 1100 equipment. The equipment was operated between room temperature and $1200^{\circ} \mathrm{C}$ with a temperature gradient of $20^{\circ} \mathrm{C} \mathrm{min}^{-1}$, using $20 \mathrm{mg}$ of sample under a static air atmosphere.

Characterization by DSC was carried out using a DSCQ200V24.4 Build 116 equipment. The following heating/cooling down/heating process was used: $r_{\text {heat }}=r_{\text {cool }}=$

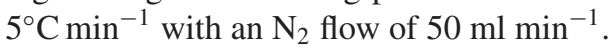

\section{Results and discussion}

\subsection{Thermogravimetric analysis and differential thermal analysis}

The TG-DTA studies revealed that the original clay presented a mass loss corresponding to the elimination of physisorbed water until approximately $120^{\circ} \mathrm{C}$ and another mass loss between 500 and $700^{\circ} \mathrm{C}$, corresponding to dehydroxylation of the layers. Both thermal events were associated with endothermic peaks observed in the DTA curves. The curves corresponding to the organoclays showed a loss in mass between room temperature and approximately $120^{\circ} \mathrm{C}$, related to the elimination of physisorbed water. Another mass loss was observed between 180 and $700^{\circ} \mathrm{C}$ and it was associated with a series of exothermic events that correspond to the phenomena of the combustion of the surfactant and dehydroxylation of the layers.

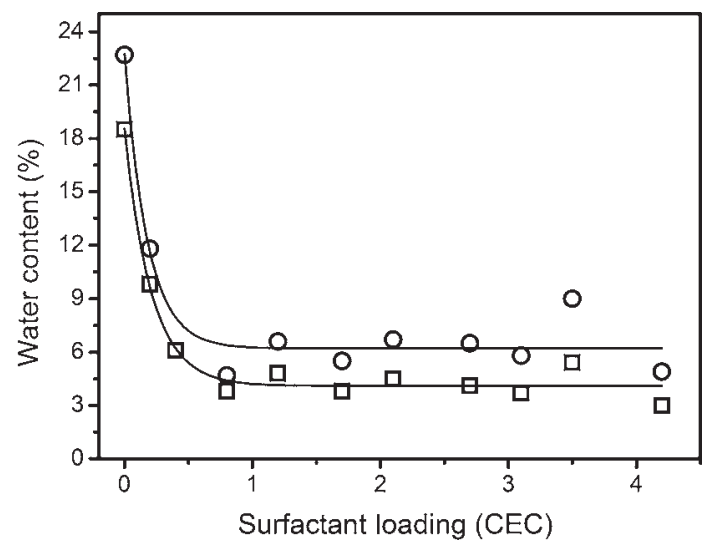

Figure 1. Physisorbed water content (\%) vs. surfactant loading. The water content is expressed as the percentage of the total mass of organoclay (square) and as the percentage of the clay mass present in each organoclay (circle). Solid lines are only for better visualization.

According to several authors, the amount of water that the organoclays absorb on the surface is related to their hydrophilicity [4-6]. To study this behaviour, the percentage of water loss can be analysed as measured in the TG studies.

The content of physisorbed water, which evaporates between room temperature and $120^{\circ} \mathrm{C}$, is shown with respect to the total mass of the organoclays (that is to say: mass of clay + mass of surfactant + mass of water) (figure 1). The percentage of clay diminished with increasing percentage of the surfactant in the organoclay. This produced a decrease in the percentage of the water content in the organoclay, which not necessarily correlates with a decrease in hydrophilicity. To eliminate this shielding effect, the percentage of physisorbed water was recalculated with regard to the mass of dry clay.

The hydrophilicity diminished with increasing SL until about 1.0 CEC and after that it remained constant. As a consequence the samples could be arranged as follows, according to their decreasing hydrophilicity:

$$
\begin{aligned}
& \text { Bent }>\text { Bent- } 0.2>\text { Bent- } 0.4>\text { Bent- } 0.8 \approx \text { Bent- } 1.0 \\
& \text { and so on }
\end{aligned}
$$

Thermal differential analysis studies reveal the thermal stability of a material, measuring the temperature that produces some kind of process that affects its stability. The higher the initial temperature of the degradation process, the more thermally stable a material will be.

Similar to the fact that a chain is as strong as the weakest of its links, thermal stability of a composite material is determined by the thermal stability of the weakest constituent. In the case of an organoclay, this is the cationic surfactant.

The samples showed a tendency towards a diminution in onset combustion temperature of the surfactant with increasing SL in the organoclays (figure 2). Values tend towards the onset combustion temperature of the pure surfactant $(97 \%$ purity). 


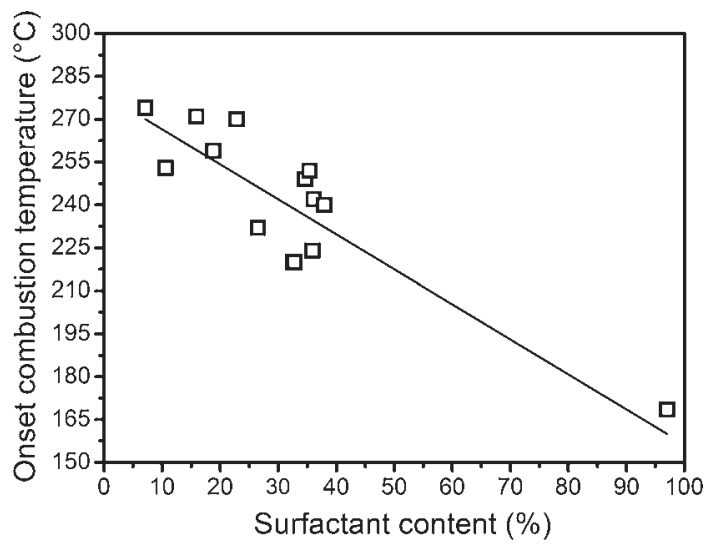

Figure 2. Onset combustion temperature of the surfactant $v s$. the surfactant loading (\%). The solid line is only for better visualization.

Previously published studies have shown that alkyl ammonium salts adsorb to smectites mainly through two mechanisms: cation exchange and Van der Waals interactions between the carbon chains of the surfactants [3,25]. At low SL, the main clay-surfactant interaction mechanism is a cation exchange. This mechanism is highly energetic and provides great stability to the surfactant, as can be observed in figure 2 . The fraction of surfactant absorbed through Van der Waals forces increases with increasing SL, thus decreasing the thermal stability and the onset combustion temperature becomes close to that of the pure surfactant.

In the DTA curves of the organoclays (figure 3 ), exothermic peaks could be observed in the range between 200 and $500^{\circ} \mathrm{C}$, which were associated with mass loss (TG is not shown). These mass losses correspond to the combustion of the surfactant. For organoclays with a SL $<1$, a peak above $300^{\circ} \mathrm{C}$ could be observed with a shoulder at $330-350^{\circ} \mathrm{C}$. In the case of organoclays with a SL $>1$, a new peak appeared between 235 and $270^{\circ} \mathrm{C}$. This reaffirms the hypothesis that the first intercalated surfactant fractions are adsorbed through strong interactions and when the CEC is exceeded, weak interactions are detected both among the surfactant molecules and between the surfactant molecules and the clay layers.

The thermal stability of the clay layers was analysed by comparing a series of endo-exothermic peaks present in the DTA curves (figure 4). These peaks are related to the structural rearrangement (SR) (crystalline transformations) of the layers as mentioned earlier and/or formation of new phases such as spinel, cristobalite or mullite [31,33,34].

One of them was an S-shaped endo-exothermic peak centred between 900 and $920^{\circ} \mathrm{C}$, which corresponds to a SR and identified as SR1. The other peak was exothermic and centred between 1150 and $1190^{\circ} \mathrm{C}$ and related to a second structural rearrangement, identified as SR2.

The temperature at which $\mathrm{SR} 1$ is produced $\left(T_{\mathrm{SR} 1}\right)$ remains constant for all SL values assayed (figure 5). This could be confirmed by the straight line adjusted to the experimental values through least square regression. The value of the

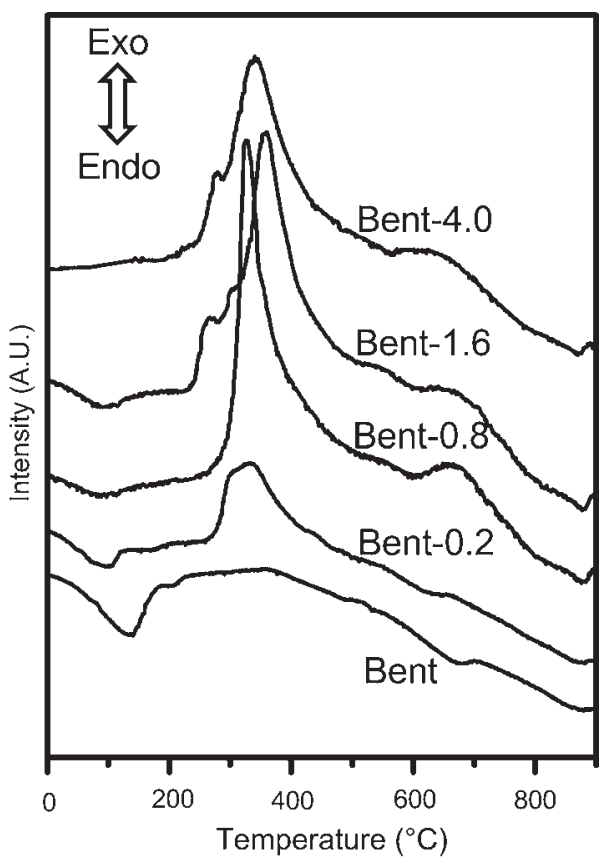

Figure 3. DTA curves of original clay and organoclays are indicated. The number of peaks increases at lower temperature when the SL becomes higher than 1 .

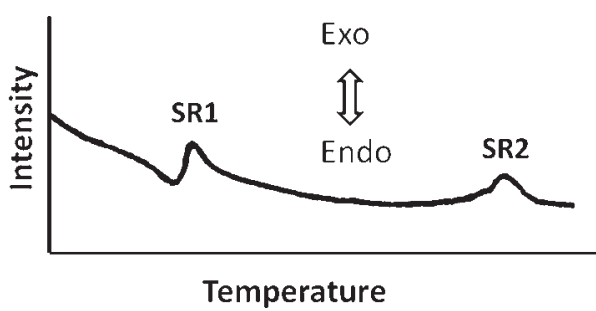

Figure 4. ATD curve showing the SR1 and SR2 thermal events.

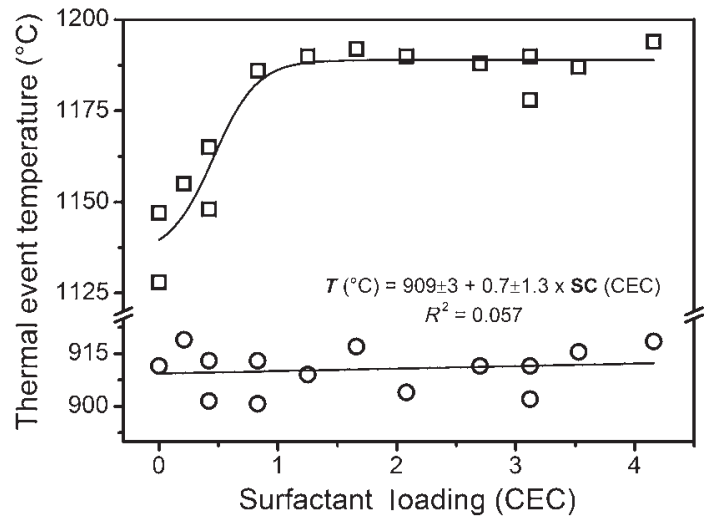

Figure 5. Structural rearrangement temperature of the clay layers as a function of the SL: SR1 (circle) and SR2 (square).

'slope \pm standard deviation' includes zero and $R_{\mathrm{RE} 1}^{2} \approx 0$, which indicates the lack of correlation between $T_{\mathrm{SR} 1}$ and the SL. In contrast, the temperature at which SR2 was produced 
$\left(T_{\mathrm{SR} 2}\right)$, increased with increasing $\mathrm{SL}$ until $\mathrm{SL} \approx 1$, after which it maintains constant (in the latter case the solid line is only for guidance).

This indicated that the two SR processes are independent because they behave differently with respect to the surfactant load. On the other hand, it could be observed that the surfactant molecules generated a structural stabilization of approximately $40^{\circ} \mathrm{C}$ in the clay layers (from 1150 to $1190^{\circ} \mathrm{C}$ ).

The surfactant molecules were eliminated through combustion at a temperature below $800^{\circ} \mathrm{C}$. Therefore, thermal stabilization was observed even though the surfactant molecules were not present in the solid material.

Under certain conditions, some small cations could be incorporated into the empty octahedral sites of the dioctahedral clays, which produce structural modifications [35]. Given this fact, it is possible that the observed structural stabilization was related to the incorporation of cations in the structure of layers, probably $\mathrm{H}^{+}$or $\mathrm{K}^{+}$, which were released during the combustion of the surfactant molecules or were part of the components of the raw clay mineral (feldspars).

\subsection{Differential scanning calorimetry}

The DSC curve of the original clay showed a sharp endothermic peak centred around $145^{\circ} \mathrm{C}$. Before the first heating cycle was completed, approximately at $170^{\circ} \mathrm{C}$, the cap of the sample holder came off probably due to the excessive pressure caused by the vapour of the water released from the clay. Therefore, it was not possible to finish the analysis of this sample.

All organoclays presented a peak between 135 and $170^{\circ} \mathrm{C}$, which is similar to the one at $145^{\circ} \mathrm{C}$ of the original clay, corresponding to the elimination of evaporated water.

The pure surfactant presented a large reversible endothermic peak during the heating process. During both heating cycles this peak was centred at $105^{\circ} \mathrm{C}$, preceded by a shoulder at $90^{\circ} \mathrm{C}$ and in the cooling down cycle it was centred around $85^{\circ} \mathrm{C}$, followed by a shoulder at $80^{\circ} \mathrm{C}$ (figure $6 \mathrm{c}$ ). There was also a second reversible peak with lower intensity, centred between 70 and $75^{\circ} \mathrm{C}$ during the heating cycles and between 65 and $70^{\circ} \mathrm{C}$ during the cooling down cycle (graph inserted in figure 6c).

The peaks corresponding to the surfactant (between 65 and $105^{\circ} \mathrm{C}$ ) and the clay (between 145 and $170^{\circ} \mathrm{C}$ ) do not overlap. This is an advantage, because it allows independent analysis of both DSC curve regions of the organoclays.

In organoclays with a SL $>1$, peaks were centred between 61 and $62^{\circ} \mathrm{C}$ and 55 and $56^{\circ} \mathrm{C}$ during the heating cycles and the cooling down cycle, respectively (figure $6 a$ and $b$ ). These peaks corresponded to transitions in the surfactant molecules ranging from highly ordered pseudo-crystalline structures to less ordered conformational structures of a liquid crystal type [36]. In other words, these transitions would correspond to a 'fusion' of the adsorbed surfactant.

The 'fusion-solidification' of the adsorbed surfactant was not observed in the DSC curves of the organoclays with a SL $<1$ (not presented in this study). The areas of the peaks

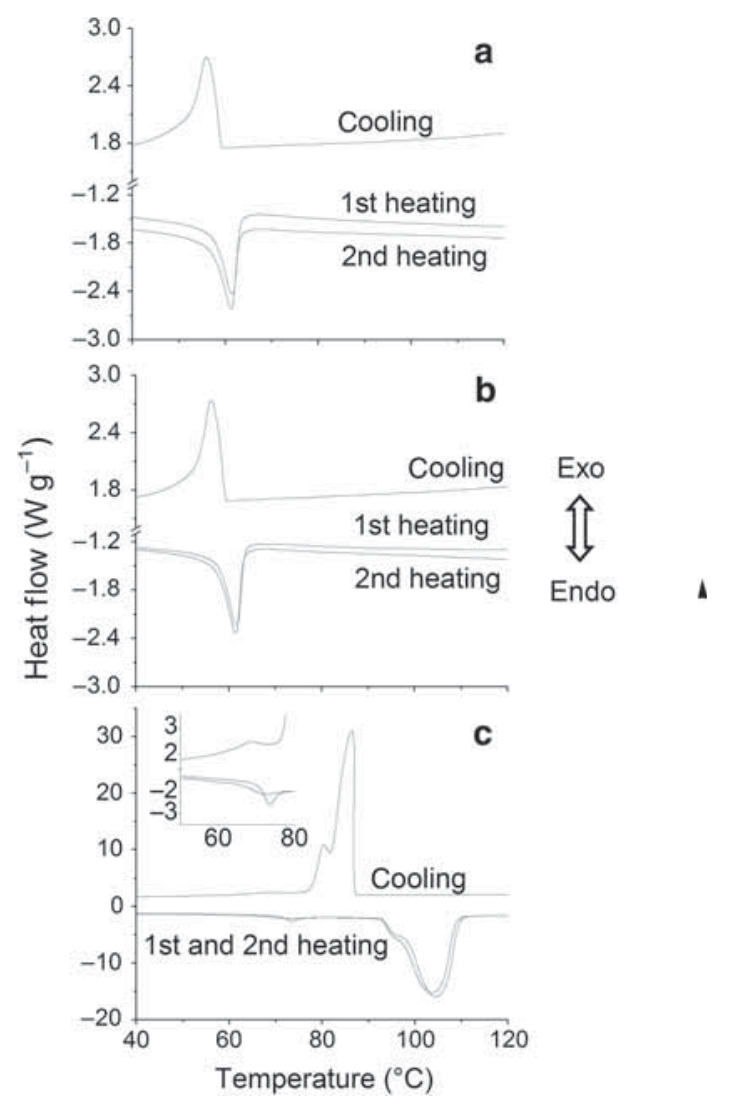

Figure 6. DSC curves in the range of $40-120^{\circ} \mathrm{C}$ of the organoclays with a SL of (a) 2.0, (b) 4.0 and (c) of the pure surfactant. The 50$80^{\circ} \mathrm{C}$ range of the pure surfactant is enlarged.

assigned to surfactant transitions, both in organoclays with SL $>1$ and in the pure surfactant, are shown in table 1 . The areas represent the heat transferred per mass unit.

The percentage of surfactant present in each organoclay (obtained through TG curves) and the amount of heat transferred during the 'fusion' of the surfactant (obtained through DSC) were compared with the pure surfactant (table 2). In the case of organoclays with a SL $>1$ the amount of heat transferred was not proportional to the surfactant mass.

According to Osman [37], when cylindrical molecules undergo an increase in temperature they gradually lose their structure, generating different mesophases. Starting from a crystalline solid state and with increasing temperature, various transition states were produced before a completely dis-ordered liquid state was reached. The temperatures at which these transitions took place could be observed through DSC, as they appear with heat transfer.

Working with organoclays prepared with mica and octadecyltrimethylammonium bromide (ODTMA) and with SL between 0.5 and 0.8 times the CEC, Osman [37] observed two endothermic peaks during the heating cycles, at 43 and $69^{\circ} \mathrm{C}$. IR and XRD techniques carried out at different temperatures attributed the first peak to a solid-solid transition between two crystalline states and the second peak to a solid-liquid transition state (fusion of the surfactant). The author proposes 
Table 1. Positions $\left({ }^{\circ} \mathrm{C}\right)$ and areas $\left(\mathrm{J} \mathrm{g}^{-1}\right)$ of peaks of DSC curves for organoclays with $\mathrm{SL}>1$ and for the pure surfactant.

\begin{tabular}{|c|c|c|c|c|c|c|c|c|}
\hline \multirow[b]{2}{*}{ Solid material } & \multicolumn{2}{|c|}{ 1st Heating cycle } & \multicolumn{2}{|c|}{ 2nd Heating cycle } & \multicolumn{2}{|c|}{ Peak area during heating cycles } & \multicolumn{2}{|c|}{ Cooling down cycle } \\
\hline & Position & Area & Position & Area & Average & Standard deviation & Position & Area \\
\hline Bent-2.0 & 61.6 & 5.1 & 61.4 & 5.1 & 5.1 & 0.01 & 55.8 & 7.1 \\
\hline Bent-4.0 & 61.8 & 6.6 & 61.6 & 6.9 & 6.8 & 0.2 & 56.1 & 8.8 \\
\hline HDTMA & 104.8 & 137.4 & 103.8 & 136.5 & 137.0 & 0.6 & 86.3 & 141.2 \\
\hline
\end{tabular}

Table 2. Mass percentages (obtained through TG) and the amount of heat transferred (obtained through DSC) of the different organoclays compared with pure surfactant.

\begin{tabular}{lcc}
\hline \multirow{2}{*}{$\begin{array}{l}\text { Solid } \\
\text { material }\end{array}$} & \multicolumn{2}{c}{ Percentages with respect to pure surfactant } \\
\cline { 2 - 3 } & Mass $(\%)$ & Heat transferred \\
\hline Bent-0.4 & 11 & 0 \\
Bent-0.8 & 16 & 0 \\
Bent-2.0 & 28 & $3.7 \%$ \\
Bent-4.0 & 36 & $5.0 \%$ \\
HDTMA & 100 & $100 \%$ \\
\hline
\end{tabular}

that the behaviour of ODTMA adsorbed to the mica by the cation exchange could be compared with that of nonadecane. Nonadecane and ODTMA presented similar solid-solid and solid-liquid transitions, although the cationic surfactant presented these transitions at temperatures between 20 and $35^{\circ} \mathrm{C}$ above that of nonadecane. According to Osman [37], this shift toward higher temperatures was caused by the fact that one of the extremes of the ODTMA was linked to the mica, which limits its translational freedom.

In this study, the pure surfactant presented three endothermic peaks during the heating stages and afterwards the corresponding exothermic peaks during the cooling down stage. At the same time, the organoclays with a SL $<1$ did not present any peaks corresponding to transition states of the surfactant, whereas samples with a SL $>1$ presented a single peak.

The peaks of the organoclays were found between 30 and $45^{\circ} \mathrm{C}$ below those of the pure surfactant during heating and cooling, respectively (table 1). This result seems contrary to findings by Osman [37], as in this study the surfactant-clay bond produced a decrease in the temperature of the peak. Osman compared the ODTMA-Mica system with that of nonadecane, while in this study a more real comparison was carried out by comparing the HDTMA-Montmorillonite with the HDTMA system.

Corroborating the results obtained with FTIR [3,25,34,38, 39], the DSC peak corresponding to the 'fusion' was not observed at low SL values because the adsorbed surfactant presented a liquid-like arrangement, i.e., a 'molten' state. On the other hand, at a SL $>1$ the adsorbed surfactant presented a solid-like arrangement and hence it showed the 'fusion' peak at the corresponding temperature in the DSC curve. Previous studies show that at a SL $>1$ the surfactant fills the mesopores of the sample as well, in addition to the surfactant intercalated at the interlayer space of the clay mineral $[3,25]$. However, it is not clear enough if the solid-like arrangement was produced at the interlayer space or inside the mesopores. Further investigations are necessary for the complete understanding of the real structure developed.

\section{Conclusions}

Organoclays with different surfactant loads were synthesized. Hydrophilicity, thermal stability and thermal stability of the original clay were assayed using thermal techniques.

Stability of the organoclays as a whole was studied by assaying the lowest temperature that produced any irreversible change in the sample detected in the DTA curves. It was found that the thermal stability of the organoclays was inversely proportional to the surfactant load.

Thermal stability of the original clay was assayed by analysing two endo-exothermic peaks characteristic of structural transformations in clay samples. It was found that the two peaks corresponded to independent structural transformations and that one of the transformations was not affected by the SL, while the presence of the surfactant stabilized the clay with regard to the structural transformation produced at about $1150^{\circ} \mathrm{C}$.

The structure of the surfactant in the interlayer space depended on the SL. At a SL $<1$ the surfactant molecules were found to be in a liquid-like environment, while at a SL $>1$ the environment was solid-like. Further studies are necessary to confirm in which place of the material the solid-like structure is developed, that is to say if the solid-like arrangement is developed only at the mesopores between the clay layers or in both places.

\section{Acknowledgements}

We gratefully acknowledge Pharm. Pablo Corregidor for their technical assistance and clarifying discussions in DSC technique. Pablo Naranjo thanks CONICET (Consejo Nacional de Investigaciones Científicas y Técnicas) for their fellowships. 


\section{References}

[1] Yürüdü C, Isci S, Ünlü C, Atici O, Ece Ö I and Güngör N 2005 Bull. Mater. Sci. 28623

[2] Rapacz-Kmita A, Stodolak-Zych E, Ziabka M, Rozycka A and Dudek M 2015 Bull. Mater. Sci. 381069

[3] Naranjo P, Molina J, Sham E L and Farfán Torres E M 2015 Quím. Nova 38166

[4] Saada A, Siffert B and Papirer E 1995 J. Colloid Interface Sci. 174185

[5] He H, Ding Z, Zhu J, Yuan P, Xi Y, Yang D et al 2005 Clays Clay Miner. $\mathbf{5 3} 287$

[6] Onal M and Sarikaya Y 2008 J. Therm. Anal. Calorim. 91 261

[7] Joshi M, Bhattacharyya A, Agarwal N and Parmar S 2012 Bull. Mater. Sci. 35933

[8] Nousir S, Platon N, Ghomari K, Sergentu A S, Shiao T C, Hersant G et al 2013 J. Colloid Interface Sci. 402215

[9] Azzouz A, Nousir S, Platon N, Ghomari K, Hersant G, Bergeron J Y et al 2013 Mater. Res. Bull. 483466

[10] Shah K J, Mishra M K, Hukla A D, Imae T and Shah D O 2013 J. Colloid Interface Sci. $\mathbf{4 0 7} 493$

[11] Burchill S, Hall P L, Harrison R, Hayes M H B, Langford J I, Livingston W R et al 1983 Clay Miner. 18373

[12] Anirudhan T S and Ramachandran M 2006 J. Colloid Interface Sci. 299116

[13] Ocampo-Perez R, Leyva-Ramos R, Mendoza-Barron J and Guerrero-Coronado R M 2011 J. Colloid Interface Sci. 364 195

[14] Kan T, Jiang X, Zhou L, Yang M, Duan M, Liu P et al 2011 Appl. Clay Sci. 54184

[15] Xin X, Si W, Yao Z, Feng R, Dua B, Yan L et al 2011 J. Colloid Interface Sci. 359499

[16] Mahadevaiah N, Vijayakumar B, Hemalatha K and Jai Prakash B S 2011 Bull. Mater. Sci. 341675

[17] Sarkar M, Dana K, Ghatak S and Banerjee A 2008 Bull. Mater. Sci. 3123
[18] Patra S K, Prusty G and Swain S 2012 Bull. Mater. Sci. 3527

[19] Choudhury T and Misra N M 2010 Bull. Mater. Sci. 33165

[20] Wang W, Li L and Xi S 1993 J. Colloid Interface Sci. 155369

[21] Vaia R A, Teukolsky R K and Giannelis E P 1994 Chem. Mater. 61017

[22] Vidal N C and Volzone C 2009 Appl. Clay Sci. 45227

[23] Zhu J, Qing Y, Wang T, Zhu R, Wei J, Tao Q et al $2011 \mathrm{~J}$. Colloid Interface Sci. $\mathbf{3 6 0} 386$

[24] Bianchi A E, Fernández M, Pantanetti M, Viña R, Torriani I, Torres Sánchez R M et al 2013 Appl. Clay Sci. 83-84 280

[25] Naranjo P M, Sham E L, Rodríguez Castellón E, Torres Sánchez R M and Farfán Torres E M 2013 Clays Clay Miner. 61 98

[26] Nayak P S and Singh B K 2007 Bull. Mater. Sci. 30235

[27] Hlavaty V and Fajnor V S 2002 J. Therm. Anal. Calorim. 67 113

[28] Lapides I, Borisover M and Yariv S 2011 J. Therm. Anal. Calorim. 10539

[29] Yariv S, Borisover M and Lapides I 2011 J. Therm. Anal. Calorim. $\mathbf{1 0 5} 897$

[30] Yariv S, Lapides I and Borisover M 2012 J. Therm. Anal. Calorim. 110385

[31] Park Y, Ayoko G A, Kristof J, Horvát E and Frost R L $2012 \mathrm{~J}$. Therm. Anal. Calorim. 1071137

[32] Bergaya F and Vayer M 1997 Appl. Clay Sci. 12275

[33] Birkenstock J, Kleemeier M, Vogt C, Wendschuh M, Hartwig A and Fischer R X 2011 Appl. Clay Sci. 54144

[34] Zhu J, He H, Zhu L, Wen X and Deng F 2005 J. Colloid Interface Sci. 286239

[35] Calvet R and Prost R 1971 Clays Clay Miner. 19175

[36] Gelfer M, Burger C, Fadeev A, Sics I, Chu B, Hsiao B S et al 2004 Langmuir 203746

[37] Osman M A, Seyfang G and Suter U W 2000 J. Phys. Chem. $B \mathbf{1 0 4} 4433$

[38] Xi Y, Frost R L and He H 2007 J. Colloid Interface Sci. 305 150

[39] Zhu L, Zhu R, Xu L and Ruan X 2007 Colloids Surf. A 30441 